\title{
A narrative review of intraperitoneal chemotherapy and cytoreductive surgery (CRS) for peritoneal metastases in gastric cancer
}

\author{
Thomas Boerner ${ }^{1}$, Pompiliu Piso ${ }^{2}$ \\ ${ }^{1}$ Department of Surgery, Memorial Sloan Kettering Cancer Center, New York, NY, USA; ${ }^{2}$ Department of Surgery, Krankenhaus Barmherzige \\ Brüder Regensburg, Regensburg, Germany \\ Contributions: (I) Conception and design: All authors; (II) Administrative support: T Boerner; (III) Provision of study materials or patients: All \\ authors; (IV) Collection and assembly of data: T Boerner; (V) Data analysis and interpretation: All authors; (VI) Manuscript writing: All authors; (VII) \\ Final approval of manuscript: All authors. \\ Correspondence to: Thomas Boerner, MD. Department of Surgery, Memorial Sloan Kettering Cancer Center, 1275 York Avenue, New York, NY \\ 10065, USA. Email: boernert@mskcc.org.
}

\begin{abstract}
Peritoneal carcinomatosis of gastric origin is an aggressive tumor entity. Historically it has been considered a terminal disease with no long-term survival, due to limited therapeutic options. However, as a better understanding of tumor biology has evolved in recent years, novel multimodal treatment strategies incorporating intraperitoneal (IP) chemotherapy-hyperthermic intraperitoneal chemotherapy (HIPEC), early postoperative intraperitoneal chemotherapy (EPIC), neoadjuvant intraperitonealsystemic chemotherapy protocol (NIPS) — and cytoreductive surgery (CRS) have demonstrated promising oncologic outcomes and even long-term survival in selected patients. Most of the studies published to date are retrospective in nature. These studies involve heterogenous patient populations, a wide variety of chemotherapeutic drugs, and show wide variation in outcomes between institutions. Thus, it is difficult to evaluate the results. This review summarizes our current knowledge regarding IP chemotherapy and CRS for peritoneal metastases (PM) in gastric cancer (GC). We describe our institutional treatment regimens. We also provide a brief overview of new, targeted therapies that may show promise in the future.
\end{abstract}

Keywords: Gastric cancer (GC); peritoneal metastases (PM); hyperthermic intraperitoneal chemotherapy (HIPEC)

Submitted Jul 16, 2020. Accepted for publication Aug 22, 2020.

doi: 10.21037/jgo-20-284

View this article at: http://dx.doi.org/10.21037/jgo-20-284

\section{Gastric cancer (GC) and peritoneal carcinomatosis-epidemiology and clinical appearance}

GC is the fifth most commonly diagnosed malignancy and the third leading cause of cancer death worldwide, with over 1 million new cases in 2018 (1). Rates are twofold higher in men than in women. In several West Asian countries it is the most commonly diagnosed cancer and the leading cause of cancer death among males (1). The disease has a dismal prognosis, especially when presenting at an advanced stage. Recurrence rates after attempted curative surgery are high: 50-60\%. One-third of local recurrence is limited exclusively to the peritoneal cavity (2). In a series of 1,178 patients with metastatic or recurrent GC, about $46 \%$ had peritoneal disease and about $30 \%$ had evidence of metastatic liver disease (3). Additionally, in about $5-20 \%$ of patients, peritoneal spread is already present at initial surgical exploration for potential curative resection $(4,5)$. In the presence of peritoneal metastases (PM) the prognosis is grim, with a median survival ranging from 1 to 13.8 months (6). Even in the absence of macroscopic PM or distant metastatic disease, malignant cells have been detected in up to $6 \%$ of peritoneal washings in patients with 
GC, despite curative resection (7). Positive cytology, even in the absence of visible peritoneal tumor, is considered to be evidence of systemic dissemination, and the outcome in these patients is similar to that of patients without PM (8).

Until recently, PM of gastric origin was regarded as a terminal disease with almost no hope of long-term survival. Response rates to systemic therapies are still very limited (9-13). In a recent meta-analysis, Thomassen et al. evaluated outcomes in a large population-based study of 5,520 patients with GC. PM was present in 706 patients (13\%), of whom 491 (9\%) presented with PM as metastatic disease only, and 215 (4\%) with PM in combination with other distant spread. Median survival in patients with PM as the only metastatic site was 4.6 months, and in patients with PM combined with other metastases the median survival was 3.3 months (9). In 2016, Boerner et al. demonstrated that multimodal treatment with gastrectomy, cytoreductive surgery (CRS), and hyperthermic intraperitoneal chemotherapy (HIPEC) is associated with improved survival for patients with PM of advanced GC, compared with matched pairs undergoing gastrectomy and palliative chemotherapy alone [17.2 months (95\% CI: 10.1-24.2) in the CRS-HIPEC group vs. 11.0 months (95\% CI: 7.4-14.6) for gastrectomy and chemotherapy alone; $\mathrm{P}=0.004]$, resulting in a two-fold increase of 2 -year survival (35.8 vs. $16.9 \%$ ) (14).

Numerous studies in the last two decades have demonstrated that the multi-modal treatment approach incorporating CRS and HIPEC may improve the prognosis for selected patients with PM from GC. This review summarizes our knowledge to date regarding intraperitoneal (IP) chemotherapy and CRS for PM in GC. We present the following article in accordance with the Narrative Review reporting checklist (available at http://dx.doi.org/10.21037/ jgo-20-284).

\section{Chemotherapeutic regimen, dosing and application}

To ensure a maximal response to HIPEC, proper selection of the chemotherapeutic drug, adequate duration of drug perfusion, and optimal temperature of the heated chemotherapeutic agent, are crucial. The most commonly used drugs in HIPEC for GC are docetaxel (15), oxaliplatin (16), cisplatin, doxorubicin and mitomycin C (17). Multiple different chemotherapeutic regimens have been used in HIPEC for GC in the past, and there is currently no consensus regarding the optimal drug regimen or dosing strategy. The duration of perfusion also varies based on the drug, ranging from 30 to 90 minutes. We currently use cisplatin $75 \mathrm{mg} / \mathrm{m}^{2}$ plus doxorubicin $15 \mathrm{mg} / \mathrm{m}^{2}$ for 90 minutes at $42{ }^{\circ} \mathrm{C}$ as our standard chemotherapeutic regimen in patients with $\mathrm{PM}$ of gastric origin.

A variety of different procedures for administering HIPEC are currently utilized. The two best known and most commonly used are the "open coliseum technique" and the "closed abdomen technique" (18). While each HIPEC perfusion technique has its own advantages and shortcomings, there is a lack of prospective controlled trials comparing the different methods of administration with respect to efficacy. Hence, as stated at the consensus meeting of the Peritoneal Surface Oncology Group International in 2006, debate continues regarding the best method for performing the HIPEC procedure. Some data suggest that the open technique is associated with an improved temperature homogeneity and better distribution of the chemotherapeutic agent in the abdominal cavity; the main drawbacks of the open technique are heat dissipation and risk of exposing the surgical team to the chemotherapy drugs. The closed abdomen technique avoids exposing the surgical team but may lead to a more uneven distribution of the drug and the associated heat (19-22). However, there is not yet sufficient scientific evidence supporting one technique over the other (20). We currently use the closed abdomen technique because, in our experience, it is a better way to keep temperatures consistently above $42{ }^{\circ} \mathrm{C}$; additionally, it provides the highest level of safety for operators and operating room personnel, as it minimizes exposure to the chemotherapeutic drug. Several experimental studies have suggested that the closed technique facilitates increased drug penetration into the tissue due to higher intraabdominal pressure $(23,24)$. The potential shortcomings of the closed technique, such as lack of direct control of the abdominal cavity, or possible suboptimal heat and chemotherapy distribution, have not been major drawbacks at our institution.

\section{Clinical studies}

Most of the data evaluating HIPEC in patients with GC are based on retrospective case-cohort studies. The first study on the effectiveness of CRS-HIPEC in the treatment of PM in GC was reported by Yonemura et al. in the late 1990s (25). This study included 83 GC patients with concurrent peritoneal dissemination who underwent CRSHIPEC with mitomycin C (30 mg), etoposide $(150 \mathrm{mg})$, 
and cisplatin $(300 \mathrm{mg})$. The reported 1 - and 5 -year overall survival (OS) rates were $43 \%$ and $11 \%$, respectively; patients who underwent complete resection survived significantly longer than those with residual disease (median OS 14.0 vs. 6.8 months; $\mathrm{P}=0.03$ ). Since then, multiple retrospective studies have demonstrated a survival benefit with the use of HIPEC for PM in GC. In a large meta-analysis including 11 randomized controlled trials and 21 non-randomized control studies, Desiderio et al. investigated the benefit of HIPEC compared with standard oncological management in GC (26). In patients with evidence of PM, an advantage in the OS rate was found in the HIPEC group at 1-year follow-up (RR: 0.67, 95\% CI: 0.52-0.86), and at 3-year follow-up similar survival rates were observed (RR: 0.99, 95\% CI: 0.93-1.06; $\mathrm{P}=0.85$ ). However, the use of HIPEC was associated with a prolonged median OS of only 4 months $(\mathrm{P}<0.001)$.

In fact, most studies have reported a minor improvement in OS of several months in patients undergoing CRSHIPEC, suggesting that only a limited, carefully selected group of patients benefit from this surgical intervention. The two most important prognostic factors for survival are the extent of peritoneal disease and the completeness of CRS $(4,27)$. A large retrospective study by Glehen et al. evaluated CRS with HIPEC (mitomycin $30-50 \mathrm{mg} / \mathrm{m}^{2} \pm$ cisplatin $50-100 \mathrm{mg} / \mathrm{m}^{2}$ for $60-120$ minutes at $41-42.5^{\circ} \mathrm{C}$ ) or postoperative IP chemotherapy [day 1 : mitomycin C $10 \mathrm{mg} / \mathrm{m}^{2}$ and days 2-5: 5-fluorouracil (5-FU) $\left.600 \mathrm{mg} / \mathrm{m}^{2}\right]$ in 159 patients with advanced GC (28). The reported median OS was 9.2 months, with 3- and 5-year survival rates of $18 \%$ and $13 \%$, respectively. The only independent prognostic factor for improved survival was completeness of cytoreduction, which led to improvement in OS to 15 months, with 3- and 5-year survival rates of $30 \%$ and $23 \%$, respectively.

Glehen et al. also showed that, in patients who had undergone complete CRS (56\%), the only prognostic factor was the extent of PM ( $\mathrm{P}=0.047)$. Despite complete cytoreduction, no patient with a Peritoneal Cancer Index (PCI) score $>19$ was alive at 6 months, and no patient with a PCI $>12$ was alive at 3 years (28). The PCI cut-off of seven was noted in a study by Yonemura et al. The authors reported that a PCI $\leq 6$ was associated with improved survival (median OS 2.8 vs. 1.1 years, $\mathrm{P}=0.0001$ ) (29). However, other investigators have shown that a PCI cutoff of 12 is associated with improved survival after CRSHIPEC compared with CRS alone. A recent meta-analysis by Coccolini et al., including nine trials and 748 patients in total, demonstrated that survival significantly changes above and below a PCI of 12 (30).

Clearly, favorable oncologic outcomes are directly related to the completeness of CRS. The evidence regarding adjuvant use of HIPEC in the multi-modal treatment approach in advanced GC was recently investigated in the CYTO-CHIP study (31). This study included 277 consecutive patients with PM from GC, who were treated with complete curative-intent CRS (no residual nodules $>2.5 \mathrm{~mm}$ ) at 19 French centers. The propensity score analysis compared patients undergoing complete CRSHIPEC with those undergoing CRS alone. Patients who underwent CRS-HIPEC showed a tremendous survival benefit of 18.8 months compared with 12.1 months for CRS alone. The 3 - and 5 -year OS rates were $26.2 \%$ and $19.9 \%$ vs. $10.8 \%$ and $6.4 \%$ (HR: 0.60; 95\% CI: $0.42-0.86$; $\mathrm{P}=0.005$ ), and 3 - and 5-year recurrence-free survival (RFS) rates were $20.4 \%$ and $17.1 \%$ vs. $5.9 \%$ and $3.8 \%(\mathrm{P}=0.001)$, respectively. These results suggest that the combination of CRS and HIPEC is essential for achieving long-term survival.

These studies have limitations, however. In addition to the limitations inherent in their retrospective nature they report a very heterogenous patient population, high variation in outcomes between institutions, and a wide variety of chemotherapeutic drugs, leading to difficulties in evaluating the results and their true clinical impact.

The only prospective randomized phase III clinical trial evaluating the impact of CRS-HIPEC in advanced GC was a study from China by Yang et al., published in 2011 (32). Sixty-eight gastric PM patients were randomized into CRS alone $(n=34)$ or CRS-HIPEC $(n=34)$. For HIPEC, cisplatin $120 \mathrm{mg}$ and mitomycin C $30 \mathrm{mg}$ each in $6,000 \mathrm{~mL}$ of normal saline was used, and administered at $43 \pm 0.5^{\circ} \mathrm{C}$ for $60-90$ minutes. The authors reported significantly improved survival in patients undergoing CRS-HIPEC versus CRS alone [11.0 months (95\% CI: 10.0-11.9) vs. 6.5 months (95\% CI: 4.8-8.2); $\mathrm{P}=0.046]$. Multivariate analysis found that CRS-HIPEC, synchronous $\mathrm{PM}$, completeness of cytoreduction (CC $0-1)$, systemic chemotherapy $\geq 6$ cycles, and no serious adverse events were independent predictors of better survival.

The results of this randomized controlled study, as well as the evidence presented in prior retrospective studies and meta-analyses, confirm that CRS-HIPEC for GC patients with PM can result in a survival benefit but should be restricted to a limited patient population based on predefined selection criteria (see Table 1). Several 
Table 1 Selection criteria for CRS-HIPEC in PM of gastric origin

Complete cytoreduction possible (CCO)

Low tumor burden $(\mathrm{PCl}<6)$

Locoregional PM (P1)

No small bowel involvement

Synchronous PM

Well-moderately differentiated tumors

No signet ring cell type

Good performance status (ECOG 0)

Prior response to systemic chemotherapy

CRS-HIPEC, cytoreductive surgery-hyperthermic intraperitoneal chemotherapy; PM, peritoneal metastases; PCI, Peritoneal Cancer Index.

prospective randomized phase II-III trials evaluating the use of HIPEC in GC are ongoing. In Germany, the effectiveness of HIPEC after CRS in GC with limited PM is currently being evaluated in a phase III study (GASTRIPEC trial) (33). The results are expected to be published by the end of 2020 and may answer some questions regarding the benefit of HIPEC.

\section{Morbidity and mortality}

The aggressive multimodal treatment approach of CRS combined with HIPEC is associated with substantial morbidity, launching further debate. In a review of 441 GC patients undergoing CRS-HIPEC, Gill et al. reported a high mortality rate of $4.8 \%$ and a morbidity rate of $21.5 \%$ (34). As systemic treatment options are still very limited in patients with advanced GC, and survival is especially dismal in the presence of PM, these mortality and morbidity rates may be tolerable. However, CRSHIPEC should only be performed in experienced centers by trained surgeons, as these factors have been shown to be directly associated with morbidity and mortality. Glehen et al. reported that the institution where the procedure was performed was an independent prognostic indicator of postoperative complications (28). It is reasonable to assume that this reflects a more rigorous patient selection by more experienced surgeons, a higher degree of surgical expertise in performing complex CRS at high-volume centers, and improved postoperative care, including management of postoperative complications. Several studies have already demonstrated a learning curve for surgeons performing
CRS-HIPEC, with improved complication rates over time (35-37). Smeenk et al. reported a series of 323 patients undergoing cytoreduction for colorectal cancer or pseudomyxoma peritonei. They showed a significant increase in the rate of complete CRS, from $35.6 \%$ to $65.1 \%$ over a period of 10 years, while the postoperative morbidity rate decreased from $71.2 \%$ to $34.1 \%$ during that same time.

\section{Neoadjuvant intraperitoneal-systemic chemotherapy protocol (NIPS), HIPEC, early postoperative intraperitoneal chemotherapy (EPIC) and normothermic intraperitoneal chemotherapy (NIPEC)}

The proportion of patients with advanced GC who receive a complete cytoreduction remains low. Even in selected patients, less than one-third obtain complete removal of all macroscopic disease (38). Therefore, the aim of new therapies is to reduce the PCI prior to CRS in order to lower the tumor burden and achieve a higher rate of complete cytoreduction, as both factors are directly linked with long-term survival. In 2006, Yonemura et al. reported a new neoadjuvant IP and systemic treatment modality for GC with peritoneal seeding: NIPS (39). The rationale for this approach was to treat advanced GC with peritoneal disease by two distinct routes: first, via systemic circulation; and second, locally, by diffusion within the peritoneal cavity, to enable a complete cytoreduction. In this study, 61 patients received bidirectional chemotherapy with IP docetaxel and carboplatin $(40 \mathrm{mg}$ docetaxel and $150 \mathrm{mg}$ carboplatin were introduced in $1,000 \mathrm{~mL}$ of saline on a weekly basis) in combination with intravenous (IV) methotrexate $\left(100 \mathrm{mg} / \mathrm{m}^{2}\right)$ and $5-\mathrm{FU}\left(600 \mathrm{mg} / \mathrm{m}^{2}\right)$. The authors reported a favorable partial response rate of $62 \%$ with consecutive complete surgical resection of macroscopic disease in one-fourth of patients. Median survival time for all patients was 14.4 months, but 20.4 months in patients with a complete cytoreduction. Based on these positive findings, a new bidirectional IP and systemic induction chemotherapy protocol was developed. The new protocol used IP (day 1) and IV (day 8) administration of docetaxel $\left(30 \mathrm{mg} / \mathrm{m}^{2}\right)$ and cisplatin $\left(30 \mathrm{mg} / \mathrm{m}^{2}\right)$ in combination with oral administration of S1 (administered for 14 days at a dose of $60 \mathrm{mg} / \mathrm{m}^{2} /$ day), followed by a 7 -day rest. This led to an even more effective eradication of peritoneal free cancer cells (PFCCs) and significant reduction in PCI score $(40,41)$. More recent clinical studies have confirmed these promising results. Using NIPS, Fujiwara et al. treated 25 GC patients 
with positive cytology or PM. The NIPS regimen consisted of IP mitomycin C $\left(20 \mathrm{mg} / \mathrm{m}^{2}\right.$ at day 1$)$ and cisplatin $\left(20 \mathrm{mg} / \mathrm{m}^{2}\right.$ at day $\left.1-5\right)$, followed by two cycles of IV triplet chemotherapy of docetaxel $\left(60 \mathrm{mg} / \mathrm{m}^{2}\right), 5-\mathrm{FU}$ $\left(350 \mathrm{mg} / \mathrm{m}^{2}\right)$ and cisplatin $\left(10 \mathrm{mg} / \mathrm{m}^{2}\right)$. The reported median OS was 27.1 months for patients with resolution of their peritoneal disease, with either negative cytology or complete regression of PM. This was significantly better than the median OS of 9.6 months for the group with persistently positive cytology or residual PM (42). In 2013, Canbay et al. reported short- and long-term outcomes in 194 GC patients with PM who received NIPS. After induction treatment, 152 of 194 patients $(78 \%)$ who showed negative results on peritoneal cytology underwent CRS-HIPEC. Complete response to NIPS was observed in $24 \%$ and complete cytoreduction was achieved in $68 \%$. Those patients with response to NIPS who underwent definitive surgery had significantly better OS than those with positive cytology or peritoneal deposits (15.8 vs. 7.5 months; $\mathrm{P}<0.001)$. Patients who obtained complete cytoreduction also had a survival of 20.5 months compared with 10.9 months for patients who underwent attempted debulking. Multivariable analysis identified pathologic response to NIPS, low tumor burden $(\mathrm{PCI} \leq 6)$, and completeness of cytoreduction as independent predictors for a better prognosis. Of note, this study also demonstrated an improvement of symptoms in patients with concurrent ascites $(n=78)$, further supporting the beneficial role of NIPS in the palliative treatment setting. However, further clinical trials are warranted to investigate this approach. Currently, our concept of the NIPS protocol is that it is appropriate in the treatment of patients with a higher volume of PM (the upper limit of PCI is not yet determined). In patients with PCI $\leq 6$, we would initially treat with upfront CRS plus HIPEC, based on the ultra-low volume of PM.

There are currently several regimens used in NIPS, but none have been compared in clinical trials.

IP chemotherapy can be delivered at different time points during treatment of advanced GC. The most frequently used time point is intraoperatively, via HIPEC. A second time point for IP drug delivery is in the early postoperative period. EPIC does not involve hyperthermia and is administered shortly after CRS, when there is minimal residual tumor burden (i.e., prior to entrapment of tumor within the fibrinous material and scar tissue that is part of the inflammatory process) (43). Finally, IP drug delivery can also be administered in the months following CRS. There is good evidence that receiving a long-term course of NIPEC after CRS may offer additional benefit to some patients after HIPEC. Even in cases of minimal residual disease after CRS and optimally performed HIPEC, the vast majority of patients develop local peritoneal recurrence (43). GC demonstrates low response rates even to newer chemotherapeutic regimens, and it is very unlikely that a single HIPEC administration will completely eradicate all GC cells. This further supports the rationale for EPIC and NIPEC.

It is important to establish that the four IP chemotherapy modalities (NIPS, HIPEC, EPIC, NIPEC) are not competitive or exclusive from one another. New treatment regimens combining CRS and perioperative chemotherapy have been developed. Recently, Yonemura et al. reported long-term outcomes after comprehensive treatment consisting of NIPS and CRS-HIPEC for GC PM (44). Among 419 patients, $63.5 \%$ achieved complete cytoreduction. For these patients, median OS was 20.9 months, with a 10 -year survival rate of $8.3 \%$. In contrast, all patients with incomplete cytoreduction died within 6 years. Future randomized controlled trials evaluating the combination of CRS and perioperative chemotherapy are clearly necessary and may provide a higher level of evidence to determine the benefit of this aggressive treatment approach.

\section{Laparoscopic HIPEC (LHIPEC)}

In the last two decades laparoscopy has been proven a safe and effective modality in the diagnosis, staging and treatment of patients with PM. In 2006, Ferron et al. first described the safety and technical feasibility of laparoscopic CRS-HIPEC in a pig model (24). In a pharmacokinetic follow-up study, they were likewise able to demonstrate that LHIPEC achieves significantly higher drug penetration of oxaliplatin from the peritoneal surface due to the higher IP pressure during laparoscopy (closed technique) versus laparotomy (open technique), probably facilitating a stronger antitumor effect (45). Yonemura et al. first reported the impact of LHIPEC in the treatment of PM from GC in humans (46). In 53 patients with peritoneal seeding from GC, two cycles of diagnostic laparoscopy and LHIPEC were performed within an interval of 1 month. At initial laparoscopy-after staging with cytologic and histologic confirmation of the diagnosis, and determination of PCI level-HIPEC was performed at $42.5-43{ }^{\circ} \mathrm{C}$ for 60 minutes using $3,000 \mathrm{~mL}$ of saline containing docetaxel $\left(30 \mathrm{mg} / \mathrm{m}^{2}\right)$ and cisplatin $\left(30 \mathrm{mg} / \mathrm{m}^{2}\right)$. At the time of the 
second laparoscopy, the amount of ascites, peritoneal cytologic status, and PCI were again determined, and the effectiveness of LHIPEC was assessed by measuring the eradication of PFCCs and the reduction of PCI between the first and the second surgery. In the LHIPEC group, 13 of the 19 patients (68\%) with positive cytology at the first surgery showed negative cytology at the second surgery, and an additional 8 patients (15\%) showed complete remission of PM and a significant reduction of PCI in the time between the two surgeries. Furthermore, involvement of the small bowel mesentery, often a limiting factor for resectability, was significantly decreased between the first and second procedures. Accordingly, LHIPEC seems to be an effective modality for the eradication of PFFCs and reduction of PCI, which are both directly associated with improved outcome at time of CRS. However, the scientific evidence is limited, and a cautious interpretation is warranted. The application of LHIPEC should be confined to experienced high-volume centers with a proven track record in both laparoscopic surgery and treatment of peritoneal surface malignancies.

\section{Future directions}

Recurrence rates in the peritoneal cavity remain high, even after curative-intent resection of advanced GC. Thus, an emerging field of interest is the prophylactic use of HIPEC to prevent future PM. Data from a prospectively collected Cancer Registry with 1,108 consecutive patients with GC undergoing radical D2 gastrectomy demonstrated that almost $50 \%$ of patients develop recurrence, with $16 \%$ manifesting metachronous PM postoperatively. Further investigation revealed that the degree of serosal involvement, nodal metastatic disease, signet cell histology and undifferentiated gradings (G3/G4) were independent risk factors for the development of metachronous PM (47). Beeharry et al. recently investigated the implications of prophylactic HIPEC with D2 radical gastrectomy for locally advanced GC in a randomized case control study (48). Eighty consecutive patients with locally advanced GC were randomly assigned to the HIPEC group (curative resection + intraoperative HIPEC with cisplatin $50 \mathrm{mg} / \mathrm{m}^{2}$ at $42.0 \pm 1.0^{\circ} \mathrm{C}$ for 60 minutes) or the control group (curative resection only). The 3 -year disease-free survival (DFS) rate analysis showed that the prophylactic HIPEC group had a significantly higher DFS rate $(93 \% v s .65 \%$, $\mathrm{P}=0.0054)$ with an associated lower peritoneal recurrence rate $(3 \%$ vs. $23 \%, \mathrm{P}<0.05)$. Currently, the GASTRICHIP trial (NCT01882933), a prospective randomized multicenter phase III trial, is open and enrolling patients. Patients with locally advanced GC (involvement of the serosa and/or lymph nodes and/or with positive cytology at peritoneal washing) are treated with perioperative systemic chemotherapy and D1-D2 curative gastrectomy with (Arm 1) or without (Arm 2) additional HIPEC. Further multicenter randomized controlled phase III trials such as the FLOT-9 are currently being planned (NCT04447352).

With genetic profiling of tumors via large scale nextgeneration sequencing, it may be possible to tailor effective drug regimens to the individual patient. This is an emerging field. Targeted therapies in the form of immunotherapy and checkpoint inhibitors have become widely available and are now important elements in multi-modal therapy for GC. It must be determined in the future if intraabdominal administration of immunotherapeutic drugs can enhance the benefit of HIPEC in PM of gastric origin. Multiple other innovative therapies to harness an immune response to cancer by IP application are under clinical investigation, including antibody-, T-cell-, and viral-based approaches (49).

\section{Summary}

HIPEC, EPIC, NIPS, and NIPEC followed by CRS have shown promising oncologic outcomes and even long-term survival in carefully selected patients with PM of gastric origin. The most commonly used drugs are docetaxel, oxaliplatin, cisplatin, doxorubicin and mitomycin C. To ensure a maximal response to HIPEC, proper drug selection, optimal temperature of the heated drug, and adequate duration of perfusion are crucial. LHIPEC has also been proven feasible. Outcomes are directly related to the completeness of CRS. These complex procedures should be performed by experienced surgeons at high-volume centers. Novel approaches, including intraabdominal administration of immunotherapeutic agents, antibody-, T-cell-, and viralbased therapies, and genetic tumor profiling using nextgeneration sequencing, are under investigation.

\section{Acknowledgments}

Funding: None.

\section{Footnote}

Provenance and Peer Review: This article was commissioned 
by the Guest Editors (Paul H. Sugarbaker and Kurt Van der Speeten) for the focused issue "Intraperitoneal Chemotherapy for Peritoneal Metastases: HIPEC, EPIC, NIPEC, PIPAC and More" published in Fournal of Gastrointestinal Oncology. This article has undergone external peer review.

Reporting Checklist: The authors have completed the Narrative Review reporting checklist. Available at http:// dx.doi.org/10.21037/jgo-20-284

Conflicts of Interest: Both authors have completed the ICMJE uniform disclosure form (available at http://dx.doi. org/10.21037/jgo-20-284). The focused issue was sponsored by the Peritoneal Surface Oncology Group International (PSOGI). The authors have no other conflicts of interest to declare.

Ethical Statement: The authors are accountable for all aspects of the work in ensuring that questions related to the accuracy or integrity of any part of the work are appropriately investigated and resolved.

Open Access Statement: This is an Open Access article distributed in accordance with the Creative Commons Attribution-NonCommercial-NoDerivs 4.0 International License (CC BY-NC-ND 4.0), which permits the noncommercial replication and distribution of the article with the strict proviso that no changes or edits are made and the original work is properly cited (including links to both the formal publication through the relevant DOI and the license). See: https://creativecommons.org/licenses/by-nc-nd/4.0/.

\section{References}

1. Bray F, Ferlay J, Soerjomataram I, et al. Global cancer statistics 2018: GLOBOCAN estimates of incidence and mortality worldwide for 36 cancers in 185 countries. CA Cancer J Clin 2018;68:394-424.

2. Yao JC, Ajani JA. Gastric cancer. Curr Opin Gastroenterol 2000;16:516-21.

3. Jo JC, Ryu MH, Koo DH, et al. Serum CA 19-9 as a prognostic factor in patients with metastatic gastric cancer. Asia Pac J Clin Oncol 2013;9:324-30.

4. Kuramoto M, Shimada S, Ikeshima S, et al. Extensive intraoperative peritoneal lavage as a standard prophylactic strategy for peritoneal recurrence in patients with gastric carcinoma. Ann Surg 2009;250:242-6.
5. Nakamura K, Ueyama T, Yao T, et al. Pathology and prognosis of gastric carcinoma. Findings in 10,000 patients who underwent primary gastrectomy. Cancer 1992;70:1030-7.

6. Saladino E, Fleres F, Mazzeo C, et al. The role of prophylactic hyperthermic intraperitoneal chemotherapy in the management of serosal involved gastric cancer. Anticancer Res 2014;34:2019-22.

7. Bentrem D, Wilton A, Mazumdar M, et al. The value of peritoneal cytology as a preoperative predictor in patients with gastric carcinoma undergoing a curative resection. Ann Surg Oncol 2005;12:347-53.

8. Leake PA, Cardoso R, Seevaratnam R, et al. A systematic review of the accuracy and utility of peritoneal cytology in patients with gastric cancer. Gastric Cancer 2012;15 Suppl 1:S27-37.

9. Thomassen I, van Gestel YR, van Ramshorst B, et al. Peritoneal carcinomatosis of gastric origin: a population based study on incidence, survival and risk factors. Int $\mathrm{J}$ Cancer 2014;134:622-8.

10. Bernards N, Creemers GJ, Nieuwenhuijzen G, et al. No improvement in median survival for patients with metastatic gastric cancer despite increased use of chemotherapy. Ann Oncol 2013;24:3056-60.

11. Janmaat VT, Steyerberg EW, van der Gaast A, et al. Palliative chemotherapy and targeted therapies for esophageal and gastroesophageal junction cancer. Cochrane Database Syst Rev 2017;11:CD004063.

12. Van Cutsem E, Moiseyenko VM, Tjulandin S, et al. Phase III study of docetaxel and cisplatin plus fluorouracil compared with cisplatin and fluorouracil as first-line therapy for advanced gastric cancer: a report of the V325 Study Group. J Clin Oncol 2006;24:4991-7.

13. Muro K, Chung HC, Shankaran V, et al. Pembrolizumab for patients with PD-L1-positive advanced gastric cancer (KEYNOTE-012): a multicentre, open-label, phase 1b trial. Lancet Oncol 2016;17:717-26.

14. Boerner T, Graichen A, Jeiter T, et al. CRS-HIPEC prolongs survival but is not curative for patients with peritoneal carcinomatosis of gastric cancer. Ann Surg Oncol 2016;23:3972-7.

15. De Simone M, Barone R, Vaira M, et al. Semi-closed hyperthermic-antiblastic peritoneal perfusion (HAPP) in the treatment of peritoneal carcinosis. J Surg Oncol 2003;82:138-40.

16. Deraco M, De Simone $M$, Rossi C, et al. An Italian Multicentric Phase II study on peritonectomy and intra peritoneal hyperthermic perfusion (IPHP) to treat patients 
with peritoneal mesothelioma. J Exp Clin Cancer Res 2003;22:41-5.

17. Kusamura S, Baratti D, Younan R, et al. The Delphi approach to attain consensus in methodology of local regional therapy for peritoneal surface malignancy. J Surg Oncol 2008;98:217-9.

18. Macrì A, Fortugno A, Saladino E. Rationale and techniques of cytoreductive surgery and peritoneal chemohyperthermia. World J Gastrointest Oncol 2011;3:169-74.

19. Elias D, Antoun S, Raynard B, et al. Treatment of peritoneal carcinomatosis using complete excision and intraperitoneal chemohyperthermia. A phase I-II study defining the best technical procedures. Chirurgie 1999;124:380-9.

20. Ortega-Deballon P, Facy O, Jambet S, et al. Which method to deliver hyperthermic intraperitoneal chemotherapy with oxaliplatin? An experimental comparison of open and closed techniques. Ann Surg Oncol 2010;17:1957-63.

21. Glehen O, Cotte E, Kusamura S, et al. Hyperthermic intraperitoneal chemotherapy: nomenclature and modalities of perfusion. J Surg Oncol 2008;98:242-6.

22. Rodríguez Silva C, Moreno Ruiz FJ, Bellido Estévez I, et al. Are there intra-operative hemodynamic differences between the Coliseum and closed HIPEC techniques in the treatment of peritoneal metastasis? A retrospective cohort study. World J Surg Oncol 2017;15:51.

23. Esquis P, Consolo D, Magnin G, et al. High intraabdominal pressure enhances the penetration and antitumor effect of intraperitoneal cisplatin on experimental peritoneal carcinomatosis. Ann Surg 2006;244:106.

24. Ferron G, Gesson-Paute A, Classe JM, et al. Feasibility of laparoscopic peritonectomy followed by intra-peritoneal chemohyperthermia: an experimental study. Gynecol Oncol 2005;99:358-61.

25. Yonemura Y, Fujimura T, Nishimura G, et al. Effects of intraoperative chemohyperthermia in patients with gastric cancer with peritoneal dissemination. Surgery 1996;119:437-44.

26. Desiderio J, Chao J, Melstrom L, et al. The 30-year experience-A meta-analysis of randomised and high-quality non-randomised studies of hyperthermic intraperitoneal chemotherapy in the treatment of gastric cancer. Eur J Cancer 2017;79:1-14.

27. Ikeguchi M, Oka A, Tsujitani S, et al. Relationship between area of serosal invasion and intraperitoneal free cancer cells in patients with gastric cancer. Anticancer Res
1994;14:2131-4.

28. Glehen O, Gilly FN, Arvieux C, et al. Peritoneal carcinomatosis from gastric cancer: a multi-institutional study of 159 patients treated by cytoreductive surgery combined with perioperative intraperitoneal chemotherapy. Ann Surg Oncol 2010;17:2370-7.

29. Yonemura Y, Elnemr A, Endou Y, et al. Multidisciplinary therapy for treatment of patients with peritoneal carcinomatosis from gastric cancer. World J Gastrointest Oncol 2010;2:85-97.

30. Coccolini F, Catena F, Glehen O, et al. Complete versus incomplete cytoreduction in peritoneal carcinosis from gastric cancer, with consideration to PCI cut-off. Systematic review and meta-analysis. Eur J Surg Oncol 2015;41:911-9.

31. Bonnot PE, Piessen G, Pocard M, et al. CYTO-CHIP: Cytoreductive surgery versus cytoreductive surgery and hyperthermic intraperitoneal chemotherapy for gastric cancer with peritoneal metastasis: a propensity-score analysis from BIG RENAPE and FREGAT working groups. J Clin Oncol 2018;36:8.

32. Yang XJ, Huang CQ, Suo T, et al. Cytoreductive surgery and hyperthermic intraperitoneal chemotherapy improves survival of patients with peritoneal carcinomatosis from gastric cancer: final results of a phase III randomized clinical trial. Ann Surg Oncol 2011;18:1575-81.

33. Rau B. Prospective multicentric phase III study. Z Gastroenterol 2014;52:262.

34. Gill RS, Al-Adra DP, Nagendran J, et al. Treatment of gastric cancer with peritoneal carcinomatosis by cytoreductive surgery and HIPEC: a systematic review of survival, mortality, and morbidity. J Surg Oncol 2011;104:692-8.

35. Smeenk RM, Verwaal VJ, Zoetmulder FA. Learning curve of combined modality treatment in peritoneal surface disease. Br J Surg 2007;94:1408-14.

36. Yan TD, Links M, Fransi S, et al. Learning curve for cytoreductive surgery and perioperative intraperitoneal chemotherapy for peritoneal surface malignancy--a journey to becoming a Nationally Funded Peritonectomy Center. Ann Surg Oncol 2007;14:2270-80.

37. Moran BJ. Decision-making and technical factors account for the learning curve in complex surgery. J Public Health (Oxf) 2006;28:375-8.

38. Yonemura Y, Fujimura T, Fushida S, et al. Hyperthermochemotherapy combined with cytoreductive surgery for the treatment of gastric cancer with peritoneal dissemination. World J Surg 1991;15:530-5. 
39. Yonemura Y, Bandou E, Sawa T, et al. Neoadjuvant treatment of gastric cancer with peritoneal dissemination. Eur J Surg Oncol 2006;32:661-5.

40. Yonemura Y, Endou Y, Sasaki T, et al. Surgical treatment for peritoneal carcinomatosis from gastric cancer. Eur J Surg Oncol 2010;36:1131-8.

41. Yonemura Y, Canbay E, Li Y, et al. A comprehensive treatment for peritoneal metastases from gastric cancer with curative intent. Eur J Surg Oncol 2016;42:1123-31.

42. Fujiwara Y, Takiguchi S, Nakajima K, et al. Neoadjuvant intraperitoneal and systemic chemotherapy for gastric cancer patients with peritoneal dissemination. Ann Surg Oncol 2011;18:3726-31.

43. Sugarbaker PH, Van der Speeten K. Surgical technology and pharmacology of hyperthermic perioperative chemotherapy. J Gastrointest Oncol 2016;7:29-44.

44. Yonemura Y, Prabhu A, Sako S, et al. Long term survival after cytoreductive surgery combined with perioperative chemotherapy in gastric cancer patients with peritoneal metastasis. Cancers (Basel) 2020;12:116.

45. Thomas F, Ferron G, Gesson-Paute A, et al. Increased tissue diffusion of oxaliplatin during laparoscopically

Cite this article as: Boerner T, Piso P. A narrative review of intraperitoneal chemotherapy and cytoreductive surgery (CRS) for peritoneal metastases in gastric cancer. J Gastrointest Oncol 2021;12(Suppl 1):S59-S67. doi: 10.21037/jgo-20-284 assisted versus open heated intraoperative intraperitoneal chemotherapy (HIPEC). Ann Surg Oncol 2008;15:3623-4.

46. Yonemura Y, Ishibashi H, Hirano M, et al. Effects of neoadjuvant laparoscopic hyperthermic intraperitoneal chemotherapy and neoadjuvant intraperitoneal/systemic chemotherapy on peritoneal metastases from gastric cancer. Ann Surg Oncol 2017;24:478-85.

47. Seyfried F, von Rahden BH, Miras AD, et al. Incidence, time course and independent risk factors for metachronous peritoneal carcinomatosis of gastric origin - a longitudinal experience from a prospectively collected database of 1108 patients. BMC Cancer 2015;15:73.

48. Beeharry MK, Zhu ZL, Liu WT, et al. Prophylactic HIPEC with radical D2 gastrectomy improves survival and peritoneal recurrence rates for locally advanced gastric cancer: personal experience from a randomized case control study. BMC Cancer 2019;19:932.

49. Beane JD, Bartlett DL. Novel Techniques and the Future of HIPEC (Immunotherapy, Viral Therapy). In: Fong Y, Gamblin TC, Han ES, et al. editors. Cancer Regional Therapy. Cham: Springer; 2020:221-34. 